\title{
TMS-EEG: From basic research to clinical applications
}

\section{Hernandez-Pavon, Julio C.}

American Institute of Physics

2014

Hernandez-Pavon , J C , Sarvas , J \& Ilmoniemi , R J 2014 , TMS-EEG: From basic research to clinical applications . in J D J Bernal-Alvarado, R Guzman-Cabrera \& M-E Brandan (eds) , 13th Mexican Symposium on Medical Physics . vol. 1626 , AIP Conference Proceedings, vol. 1626 , American Institute of Physics, NY , pp. 15-21, Mexican Symposium on Medical Physics, León, Guanajuato , Mexico , 15/03/2014 . https://doi.org/10.1063/1.4901355

http://hdl.handle.net/10138/152855

https://doi.org/10.1063/1.4901355

publishedVersion

Downloaded from Helda, University of Helsinki institutional repository.

This is an electronic reprint of the original article.

This reprint may differ from the original in pagination and typographic detail.

Please cite the original version. 


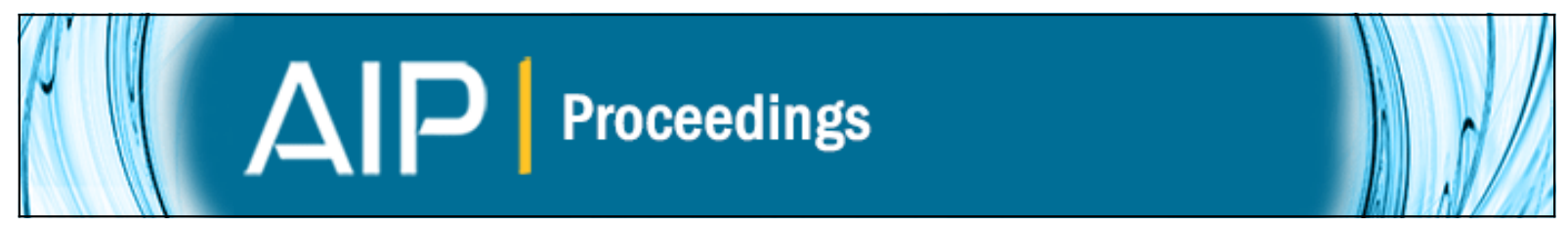

\section{TMS-EEG: From basic research to clinical applications}

Julio C. Hernandez-Pavon, Jukka Sarvas, and Risto J. Ilmoniemi

Citation: AIP Conference Proceedings 1626, 15 (2014); doi: 10.1063/1.4901355

View online: http://dx.doi.org/10.1063/1.4901355

View Table of Contents: http://scitation.aip.org/content/aip/proceeding/aipcp/1626?ver=pdfcov

Published by the AIP Publishing

\section{Articles you may be interested in}

Transforming ultrasound basic research in to clinical systems

J. Acoust. Soc. Am. 136, 2220 (2014); 10.1121/1.4900059

Clinical Ion Beam Applications: Basic Properties, Application, Quality Control, Planning

AIP Conf. Proc. 1099, 439 (2009); 10.1063/1.3120069

Extracorporeal shock wave therapy in orthopedics, basic research, and clinical implications

J. Acoust. Soc. Am. 117, 2369 (2005); 10.1121/1.4785605

Biomagnetism of the Fetus and Adult: Recent Basic and Clinical Applications

AIP Conf. Proc. 630, 79 (2002); 10.1063/1.3682848

Hadron-induced reactions: From basic research to new technological applications

AIP Conf. Proc. 392, 325 (1997); 10.1063/1.52476 


\title{
TMS-EEG: From Basic Research to Clinical Applications
}

\author{
Julio C. Hernandez-Pavon ${ }^{1,2, \text { a) }}$, Jukka Sarvas ${ }^{1}$, Risto J. Ilmoniemi ${ }^{1,2}$ \\ ${ }^{1}$ Department of Biomedical Engineering and Computational Science (BECS), Aalto University School of Science, \\ Espoo, Finland \\ ${ }^{2}$ BioMag Laboratory, HUS Medical Imaging Center, Helsinki University Central Hospital, Helsinki, Finland

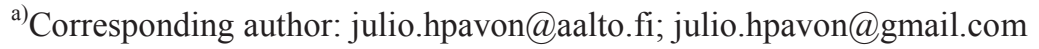

\begin{abstract}
Transcranial magnetic stimulation (TMS) combined with electroencephalography (EEG) is a powerful technique for non-invasively studying cortical excitability and connectivity. The combination of TMS and EEG has widely been used to perform basic research and recently has gained importance in different clinical applications. In this paper, we will describe the physical and biological principles of TMS-EEG and different applications in basic research and clinical applications. We will present methods based on independent component analysis (ICA) for studying the TMS-evoked EEG responses. These methods have the capability to remove and suppress large artifacts, making it feasible, for instance, to study language areas with TMS-EEG. We will discuss the different applications and limitations of TMS and TMS-EEG in clinical applications. Potential applications of TMS are presented, for instance in neurosurgical planning, depression and other neurological disorders. Advantages and disadvantages of TMS-EEG and its variants such as repetitive TMS (rTMS) are discussed in comparison to other brain stimulation and neuroimaging techniques. Finally, challenges that researchers face when using this technique will be summarized.
\end{abstract}

\section{INTRODUCTION}

Transcranial magnetic stimulation (TMS) has been used for more than 25 years to non-invasively stimulate the human brain. TMS is a method for stimulating the brain through the intact scalp with a strong magnetic field in order to produce neuronal activity [1]. When TMS is combined with electroencephalography (EEG), one can study cortical excitability and functional connectivity between different areas of the brain [2]. There are different modalities of TMS: single-pulse TMS, rTMS, paired-pulse TMS, etc. When a TMS pulse is delivered, it does not only affect the neuronal activity in the stimulated area but activates/modulates also the excitability of interconnected distant cortical areas [3]. TMS activates neurons in the cortex, rather than deep parts of the corticospinal tract. When TMS is delivered to the primary motor cortex (M1), cortico-spinal motorneurons are activated inducing muscle evoked potentials (MEP).

The causality produced by TMS opens up unique possibilities to map the human brain, which can be done at the system level of sensory, cognitive, and motor brain networks. Therefore, combining TMS with various neuroimaging techniques allows one to investigate human brain functions. The different neuroimaging techniques offer complementary information and have different methodological strengths and weaknesses.

In the first part of this paper, we describe the basics of TMS. In the second part, we present the combination of TMS and neuroimaging techniques; we will focus on TMS combined with EEG. We will discuss some methods to study TMS-evoked EEG data. Finally, we discuss the potential application of TMS in clinical applications.

XIII Mexican Symposium on Medical Physics

AIP Conf. Proc. 1626, 15-21 (2014); doi: 10.1063/1.4901355

(C) 2014 AIP Publishing LLC 978-0-7354-1263-7/\$30.00 


\section{PHYSICAL AND BIOLOGICAL PRINCIPLES OF TMS}

The idea of TMS is based on electromagnetic induction, described by Faraday's law, where an electric current in a primary circuit (coil) produces a magnetic field B, and the time-varying magnetic field induces an electric field $\mathrm{E}$ and therefore, a current flow (eddy currents) in nearby conductors (brain). The chain of events in TMS is described in Fig. 1. Consequently, E produces neuronal activation. When B changes slowly or is static, no neuronal excitation occurs. The strength of $\mathbf{B}$ in TMS is of the order of $2 \mathrm{~T}$. The spatial resolution varies from several mm up to centimeters, depending on the coil, TMS intensity and target area. TMS stimulates superficial areas more strongly than deep areas. The temporal resolution of TMS is sub-milliseconds, which allows for real-time modulation of the brain. The electric field strength for brain stimulation should usually be of the order of $30-100 \mathrm{~V} / \mathrm{m}$ to elicit significant neuronal activation [4].

\section{Time-varying magnetic field $\Rightarrow$ Electric field $\Rightarrow$ Neuronal activation}

FIGURE 1. Diagram of events during TMS. The time-varying magnetic field $\mathbf{B}$ induces an electric field $\mathbf{E}$ on the brain. The electric field produces movement of ions in pyramidal neurons, leading to depolarization of membranes and subsequent neuronal activation.

The distribution of the electric field induced in the brain depends on the shape of the coil, the location and orientation of the coil with respect to the tissue and the electrical conductivity of the tissue. At the microscopic level, TMS forces free charges in intra and extracellular volumes to move by the electric field. The magnetic field B has no essential direct effect on ions; the effect is produced by the electric field $\mathbf{E}$, which affects the drift on ions and orients dipoles.

When one stimulus is applied at a time, it refers to single-pulse TMS. The rate of these pulses is lower than $1 \mathrm{~Hz}$. Most knowledge about the effects of TMS on the human cortex comes from studies performed on the primary motor cortex. Stimulation of this area evokes activity in muscles on the opposite side of the body, which can be measured by using electrophysiological methods such as electromyography (EMG). In contrast, stimulation of most other parts of the cortex (at least with single pulses) has no obvious effects. One exception is the stimulation of visual cortex, which can elicit phosphenes.

TMS of the motor cortex exhibits two effects that are likely to happen when stimulating other cortical areas. The size of the response depends, first, on the level of activity in the cortex at the time the stimulus is given and, second, on the orientation of the TMS coil on the head [5]. The orientation of the coil plays an important role in the response since neurons on the cortex are oriented either perpendicular or parallel to the surface. Depending on the folding of the cortex, the neurons have specific orientations with respect to the TMS-induced currents that will favor one or another population. As a consequence of this fact, the activation of the hand area of the motor cortex occurs at the lowest threshold when the stimulus induces posterior-to-anterior currents in the brain along a line perpendicular to the length of the central sulcus.

When a train of pulses is delivered, the TMS technique is called repetitive TMS or rTMS. Low-frequency rTMS refers to a train of pulses at frequencies of $\mathrm{f}<1 \mathrm{~Hz}$ that tends to have an inhibitory effect, whereas high-frequency rTMS refers to a train of pulses delivered at frequencies $\mathrm{f}>5 \mathrm{~Hz}$ and usually has been found to have an excitatory effect [6]. Due to the outlasting effects of rTMS, this technique has generated a lot of interest in studying cognitive processes and as a potential therapeutic tool for the treatment of conditions such as stroke (motor recovery, dysphagia, aphasia), Parkinson's disease (bradykinesia, dyskinesia), Alzheimer's disease, schizophrenia, depression, and pain $[6,7]$. 


\section{TMS COMBINED WITH NEUROIMAGING TECHNIQUES}

In recent years, the use of TMS combined with a variety of different neuroimaging techniques has greatly increased. The spatio-temporal responses produced by TMS can be mapped by using different neuroimaging techniques. TMS has been used with computed tomography (CT) and magnetic resonance imaging (MRI) to relate its effect to the structural properties of the brain, whereas the hemodynamic responses have been studied with functional magnetic resonance imaging (fMRI), near infrared spectroscopy (NIRS) and positron emission tomography (PET); electromagnetic responses have been assessed by using EEG and magnetoencephalography (MEG), see [8] for a review.

In general, TMS can be combined with other techniques in two ways; online (when TMS is applied while neuroimaging is being performed) or offline (when TMS is applied before or after neuroimaging). The first approach allows studying the immediate effects of TMS on neuronal activity, while the second one allows studying the lasting functional effects of TMS. Undoubtedly, it is more demanding to perform studies online; the degree of difficulty depends on the neuroimaging technique. So far, major progress has been made in combining TMS with fMRI, NIRS and EEG. TMS combined with EEG is the only combination that allows getting millisecond time resolution, which allows one to measure the immediate cortical responses to TMS. TMS with EEG can be combined in both ways, i.e., online and offline. However, it is more beneficial to perform studies online.

TMS combined with EEG allows one to non-invasively study functional connectivity, and cortical excitability of different areas of the brain [9]. The first study on TMS-EEG was reported in 1997 [2]. Nevertheless, to combine TMS with simultaneous EEG is challenging, the main problem being that the strong magnetic field induces currents in the electrode leads, which produces saturation of the amplifiers and creates artifacts. This problem can be addressed by using gain-control and sample-and-hold circuits to block the artifacts induced by TMS in the leads [10]. Besides that, during the application of the TMS pulse, some current can pass through the electrode-electrolyte interface, causing polarization, which produces an EEG baseline shift that can last for hundreds of milliseconds. Overheating of the electrodes is another problem that may occur, particularly when long trains of pulses are delivered. Those problems can be effectively solved by using special electrodes, such as small $\mathrm{Ag} / \mathrm{AgCl}$ electrodes $[10,11]$.

Despite the fact that the current TMS-EEG systems have been technically improved to avoid the problems previously described, there are still some difficulties in stimulating certain regions of the brain. If TMS is applied over facial nerves or lateral areas close to cranial muscles, large biological muscle artifacts lasting for tens of milliseconds are activated, which mask the evoked EEG signals [12].

\section{TMS-EVOKED EEG DATA}

TMS evokes different electrical responses according to the stimulation site. Figure 2 shows the averaged response in a single channel near the stimulation of the left M1. The EEG response to a single TMS pulse is composed of several deflections, which are named according to their polarity and latency. For example; N15 (negative deflection about $15 \mathrm{~ms}$ post-stimulus) is related with the activation of the premotor cortex and N100 (negative deflection about $100 \mathrm{~ms}$ ) is associated with inhibitory responses. However, those responses vary slightly between subjects, experimental setup, and stimulation conditions.

Figure 3 shows typical EEG recordings after stimulating the motor cortex (M1), dorsal premotor cortex (dPMC), and Broca's area (BA). Those signals correspond to the responses recorded with electrodes near the respective stimulation sites. When BA and APMC are stimulated with TMS, the amplitude of the EEG recordings is much larger than the brain signals. The brain signals represent neurophysiological activity and have amplitude smaller than $\pm 30 \mu \mathrm{V}$, see Fig. 2. The amplitude of the artifacts in BA is about $2500 \mu \mathrm{V}$. As a consequence, the artifacts that arise after stimulating lateral brain areas mask the brain signals because they can be orders of magnitude larger than the brain signals and last for tens of milliseconds [12]. 


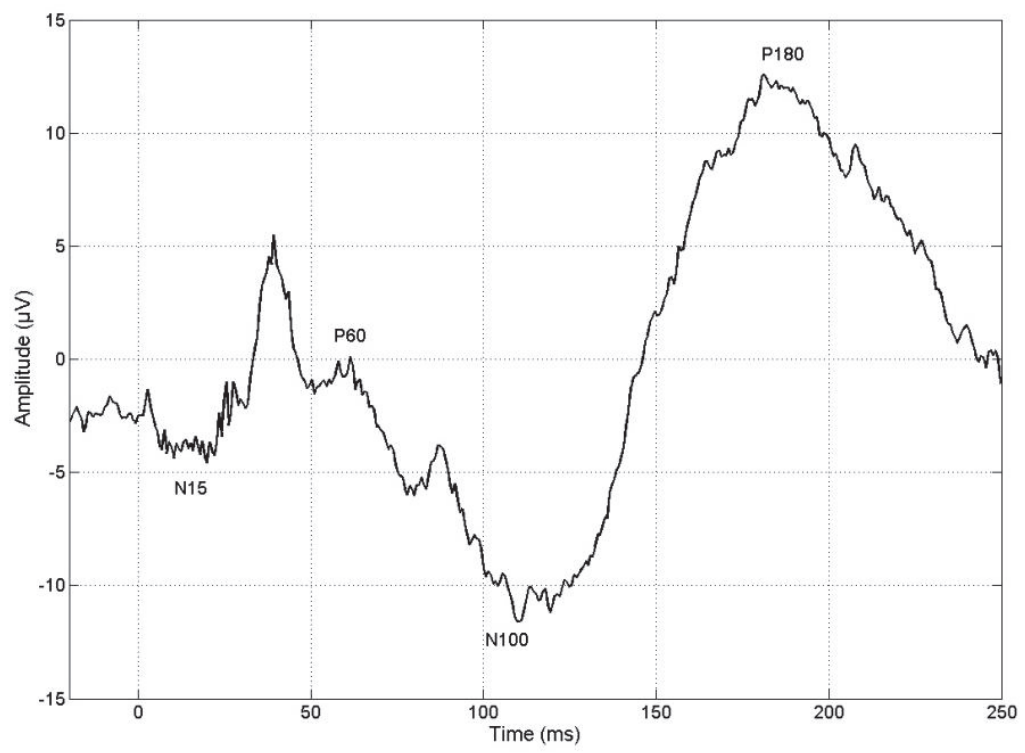

FIGURE 2. Typical averaged TMS-evoked EEG response from the motor cortex (M1) in a single channel near the stimulation site. The deflections are named according to their polarities and latencies.
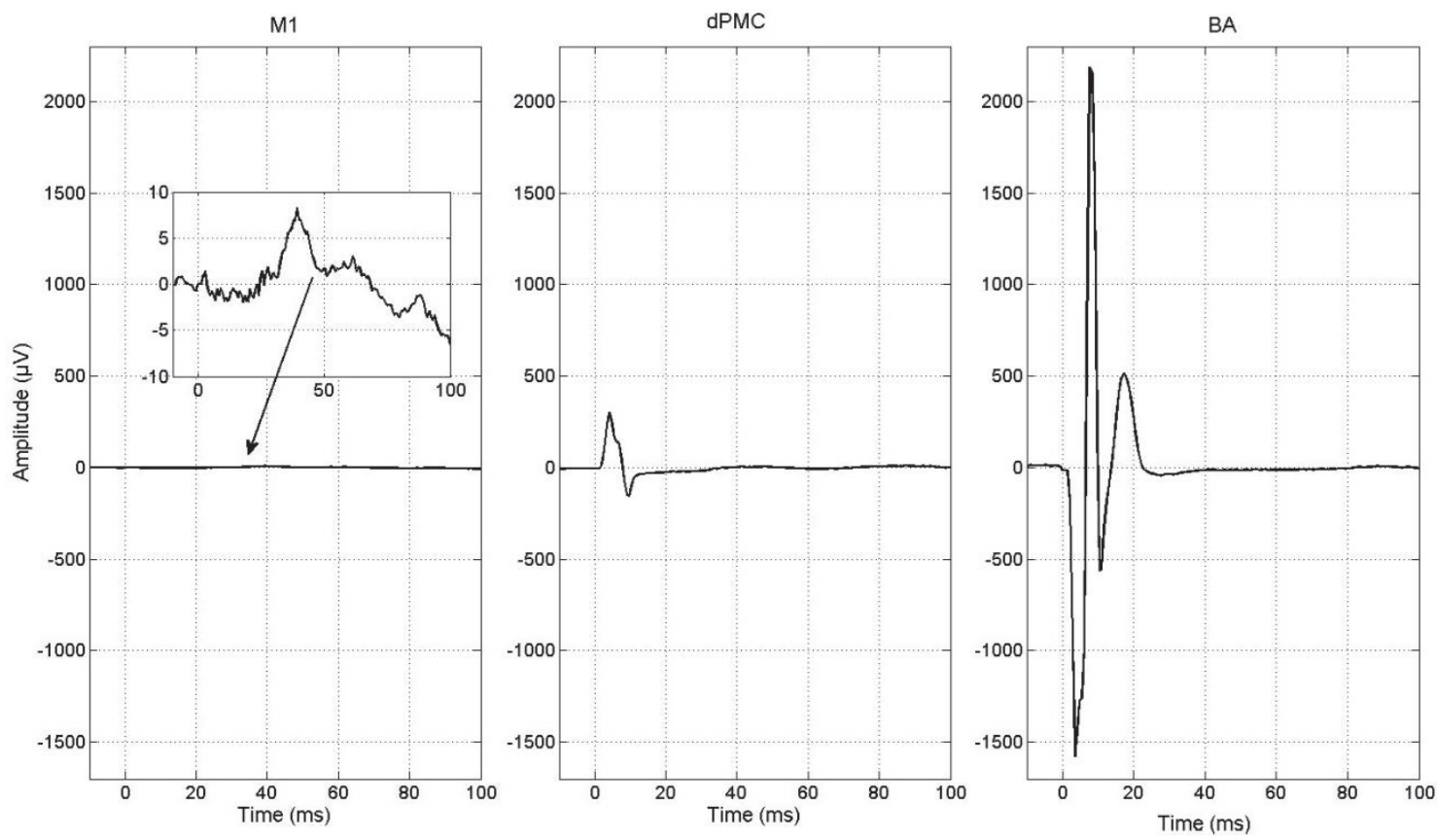

FIGURE 3. Typical waveforms after stimulating the motor cortex (M1), dorsal premotor cortex (dPMC), and Broca's area (BA) in a representative subject. The artifacts in both $\mathrm{APMC}$, and BA are much larger than the brain signals, which have amplitudes that are much smaller than the size of the artifacts; see the response of M1.

It is evident when lateral areas are stimulated with TMS, it is not possible to extract useful neurophysiological information from the recorded responses because of the large artifacts. This makes it impossible to either study the spread of neuronal activity and connectivity in lateral areas of the brain. Hence, effective methods should be found to solve this problem. 
A good technique to remove the muscle artifacts from the TMS-evoked EEG data is to use methods based on independent component analysis (ICA). For instance, stimulation of BA evokes strong muscle artifacts that contaminate the EEG recordings. In fact, the responses recorded by the electrodes are composed of mixed signals, i.e., brain signals and muscle artifacts. ICA is a powerful method to separate the EEG signals into artifactual and brain components. ICA has been extensively used in removing artifacts in EEG and MEG recordings [13]. Figure 4 shows the results after removing the artifactual components by ICA. The highest amplitude of the artifacts was 3000 $\mu \mathrm{V}$ while the highest brain signal amplitude was about $25 \mu \mathrm{V}$. The artifactual signals presented different deflections, one of them between 2 and $10 \mathrm{~ms}$ and other at about $15-30 \mathrm{~ms}$; with ICA the data was free of artifacts after $60 \mathrm{~ms}$. For more details of the methods, see [12, 14].

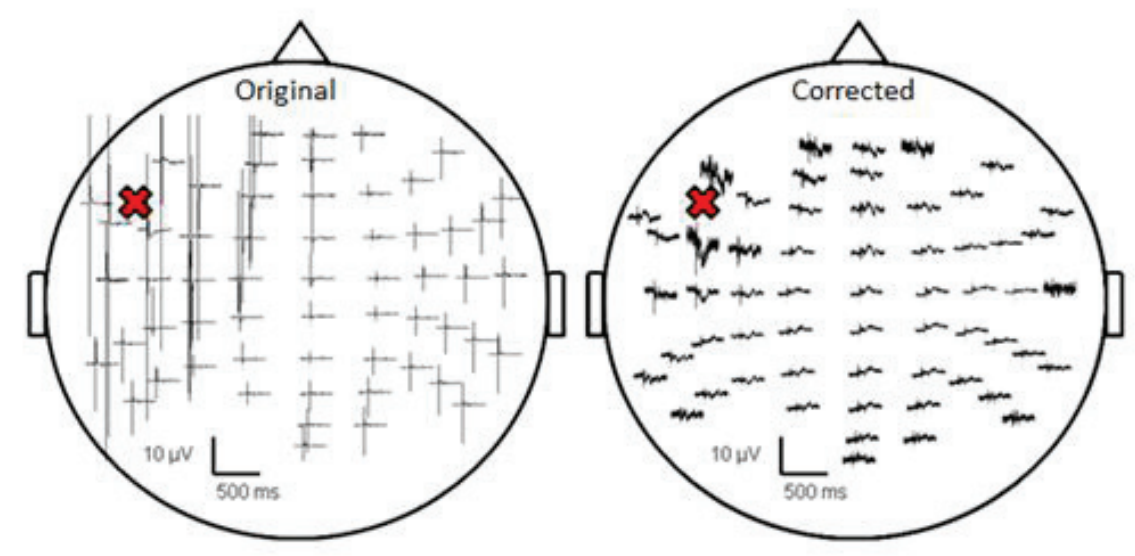

FIGURE 4. BA data before and after removing the artifactual components with ICA.

\section{CLINICAL APPLICATIONS OF TMS-EEG}

It has been a big effort to use TMS in clinical applications. Nevertheless, TMS is under active study in the treatment of a range of psychiatric and neurological disorders. The mechanisms by which TMS could exert lasting effects in illnesses subserved by distributed neuronal networks are at present largely unknown. In this section, we list only some examples where TMS has been used for clinical purposes. Treatment of depression was the first major therapeutic goal set for TMS. Trains of rTMS at $5-20 \mathrm{~Hz}$ are usually delivered to the dorsolateral prefrontal cortex (DLPFC) to treat depression and the use of TMS for treating depression was approved by the Food and Drug Administration (FDA) in 2008 [15,16]. In addition, there is new evidence that stimulation of other areas such as dorsomedial prefrontal cortex (DMPFC), frontopolar cortex (FPC), ventromedial prefrontal cortex (VMPFC), and ventrolateral prefrontal cortex (VLPFC) may improve the treatment of depression [17]. However, much more research is needed, particularly with issues such as the TMS coil location, stimulation intensity and frequency, and dosing strategy.

One of the earliest and most successful applications of inhibitory, low-frequency rTMS was to treat schizophrenia-related auditory hallucinations. Functional neuroimaging shows activity in the temporal cortex during auditory hallucinations, providing an accessible target and a compelling physiological rationale for a treatment producing local neural depression [18]. It has been shown that rTMS at $1 \mathrm{~Hz}$ targeted to the left temporoparietal area suppressed auditory hallucinations [19]. Similarly to schizophrenic auditory hallucinations, tinnitus is associated with activity in temporoparietal cortex in functional imaging studies, providing a similar target and rationale for intervention [18].

In the case of Parkinson disease (PD), neurophysiological models indicate decreased excitatory drive on the motor cortex from the basal ganglia, via thalamic relays. Consequently, this has led to the rationale for directing 
high-frequency rTMS to the motor cortex in PD patients [18]. It is important to keep in mind that even though TMS is a promising tool for therapeutic purposes, there are many scientific gaps that restrict its use in clinical applications, therefore more studies should be carried out.

\section{CONCLUSIONS}

In the last 28 years, during which TMS has been available, the number of studies on basic research and clinical applications has increased considerably. TMS has been used to study basic neuroscience, obtain diagnostic information, perform presurgical evaluation, treat different disorders of the brain, and map the brain. Nevertheless, there are many open questions basically when TMS is used in clinical studies. TMS combined with EEG represents a powerful technique for directly and non-invasively assessing cortical excitability and connectivity in humans. In this paper, we have described different studies where TMS-EEG has been used in basic research and clinical conditions. Nevertheless, more studies with TMS-EEG should be carried out to explain functional and effective connectivity, to describe the state of the cortex, and map the brain. In addition, methods for analyzing TMS-evoked EEG data and source analysis should be implemented. The knowledge generated from basic research will help to apply TMS in clinical applications (Fig. 5).

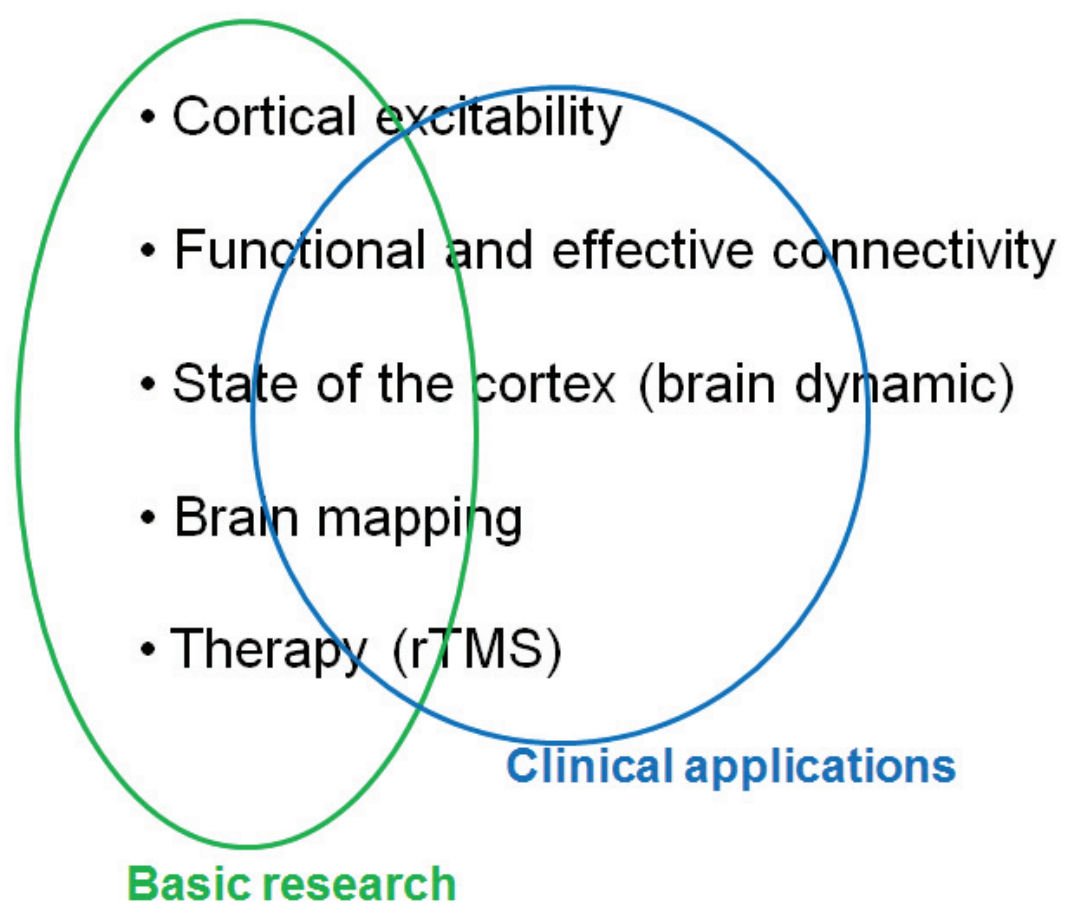

FIGURE 5. Diagram that shows the relation between basic research and clinical application of TMS-EEG. 


\section{ACKNOWLEDGMENTS}

The authors want to thank the Academy of Finland for funding. J.C. Hernandez-Pavon also wants to thank CONACYT (Consejo Nacional de Ciencia y Tecnologia) Mexico for support.

\section{REFERENCES}

1. A.T. Barker, R. Jalinous, I.L. Freeston. Lancet 1, 1106-1107 (1985).

2. R.J. Ilmoniemi, J. Virtanen, J. Ruohonen, J. Karhu, H.J. Aronen, R. Näätänen, T. Katila. Neuroreport 8, 35373540 (1997).

3. H. Siebner, M. Peller, P. Bartenstein, F. Willoch, C. Rossmeier, M. Schwaiger, B. Conrad. Human Brain Mapping 12, 157-167 (2001).

4. R.J. Ilmoniemi, J. Ruohonen, J. Karhu. Critical reviews in Biomedical Engineering 27, 241-284 (1999).

5. M.C. Ridding, J.C. Rothwell. Nature Review Neuroscience 8, 559-567 (2007).

6. T. Platz, J.C. Rothwell. Restorative Neurology and Neuroscience 28, 387-398 (2010).

7. E.M. Wassermann, S.H. Linsanby. Clinical Neurophysiology 112, 1367-1377 (2001).

8. H.R. Siebner, T.O. Bergmann, S. Bestmann, M. Massimini, H. Johansen-Berg, H. Mochizuki, D.E. Bohning, E.D. Boormann, S. Groppa, C. Miniussi, A. Pascual-Leone, R. Huber, P.C.J. Taylor, R.J. Ilmoniemi, et al., Brain Stimulation 2, 58-80 (2009).

9. R.J. Ilmoniemi, D. Kičić. Brain Topography 22, 233-248 (2010).

10. J. Virtanen, J. Ruohonen, R. Näätänen, R.J. Ilmoniemi. Medical \& Biological Engineering \& Computing 37, 322-326 (1997).

11. J.R. Ives, A. Rotenberg, R. Poma, G. Thut, A. Pascual-Leone. Clinical Neurophysiology 117, 1870-1875 (2006).

12. R.J. Korhonen, J.C. Hernandez-Pavon, J. Metsomaa, H. Mäki, R.J. Ilmoniemi, J. Sarvas. Medical \& Biological Engineering \& Computing 49, 397-407 (2011).

13. J. Onton, M. Westerfield, J. Townsend, S. Makeig. Neuroscience \& Biobehavioral Reviews 30, 808-822 (2006).

14. J.C. Hernandez-Pavon, J. Metsomaa, T. Mutanen, M. Stenroos, H. Mäki, R.J. Ilmoniemi, J. Sarvas. Journal of Neuroscience Methods 209, 144-157 (2012).

15. P.B. Fitzgerald, Z.J. Daskalakis. Brain Stimulation 5, 287-296 (2012).

16. M.S. George, S.H. Lisanby, D. Avery, W.M. McDonald, V. Durkalski, M. Pavlicova, B. Anderson, Z. Nahas. P. Zarkowski, P.E. Holtzheimer, T. Schwartz, H.A. Sackeim. Archives of General Psychiatry 67, 507-516 (2010).

17. J. Downar, Z.J. Daskalakis. Brain Stimulation 6, 231-240 (2013).

18. E.M Wassermann, T. Zimmermann. Pharmacology \& Therapeutics 133, 98-107 (2012).

19. R.E. Hoffman, R. Gueorguieva, K.A. Hawkins, M. Varanko, N.N. Boutros, Y.T. Wu, K. Carroll, J.H. Krystal. Biological Psychiatry 58, 97-104 (2005). 